Musées, Patrimoine et Culture scientifiques et techniques

$133 \mid 2011$

janvier - février 2011

\title{
Musées et seniors : chronique d'une rencontre annoncée
}

Museum and Senior citizens: chronic of an announced meeting

Jean-Luc Bourges

\section{OpenEdition}

Journals

Édition électronique

URL : http://journals.openedition.org/ocim/657

DOI : $10.4000 /$ ocim. 657

ISSN : 2108-646X

Éditeur

OCIM

Édition imprimée

Date de publication : 1 janvier 2011

Pagination : 24-30

ISSN : 0994-1908

\section{Référence électronique}

Jean-Luc Bourges, « Musées et seniors : chronique d'une rencontre annoncée », La Lettre de l'OCIM [En ligne], 133 | 2011, mis en ligne le 01 janvier 2013, consulté le 10 décembre 2020. URL : http:// journals.openedition.org/ocim/657 ; DOI : https://doi.org/10.4000/ocim.657 


\title{
Musées et seniors : chronique d'une rencontre annoncée
}

\author{
Jean-Luc Bourges *
}

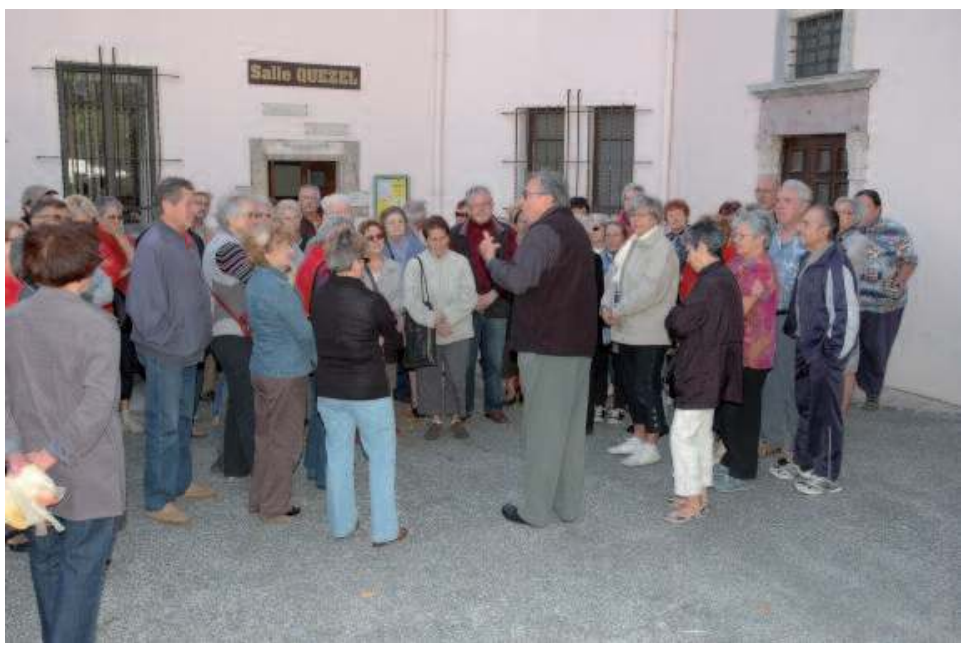

L'intérêt des seniors pour le Patrimoine n'est plus à démontrer tout comme celui pour les visites en groupe. (c) Ville historique de Saint-Pons-de-Thomières/Michel Coroir
* Jean-Luc Bourges est consultant en muséologie bourges.consultant@gmail.com
À partir d'études statistiques et d'enquêtes menées sur les pratiques muséales des seniors et leurs attentes en la matière, l'auteur met en lumière une série d'adaptations indispensables pour l'institution muséale, dressant ainsi une sorte de vade-mecum à l'usage des musées pour l'appréhension de ce public spécifique.

Pendant des décennies, les sociétés occidentales ont oublié une catégorie qui aujourd'hui revient sur le devant de la scène : les seniors. Chaque année, l'idole du « jeunisme » perd de son aura et semble céder la place à une reconnaissance intrinsèque des valeurs attachées à chaque groupe d'âge. L'arrivée des baby-boomers dans la catégorie des seniors contribue à modifier les données, en remettant en cause de nombreux clichés et stéréotypes. Il n'en demeure pas moins que les institutions culturelles et plus particulièrement les musées s'intéressent encore peu à cette classe d'âge, peut-être par habitude ou par oubli, sûrement par manque de moyens, très certainement par méconnaissance. Les études touchant les rapports entre les seniors et les musées sont en effet particulièrement rares de ce côté de l'Atlantique alors qu'elles ont tendance à l'être de moins en moins en Amérique du Nord.

\section{Préalables à la rencontre}

Caractères généraux de ce nouveau public Il s'agit tout d'abord d'un public difficile à définir et à appréhender. Les seniors sont en effet loin de constituer un groupe homogène et les considérer 
comme tels serait une erreur. L'entrée dans cette catégorie ne saurait être rapportée à une question d'âge, mais plutôt à un changement comportemental. En se référant à la pyramide d'Abraham Maslow (1), les seniors se caractérisent avant tout par d'importants besoins d'auto-accomplissement, à savoir ceux de se réaliser, d'exploiter et de mettre en valeur un potentiel personnel dans tous les domaines de la vie.

Dans cette courte étude, on considèrera les seniors comme un terme générique pour désigner les personnes âgées de plus de 60 ans, souvent à la retraite ou à la veille de l'être, et de moins de 80 ans. Pourquoi un âge limite? Parce que jusqu'à cet âge, les seniors se déplacent encore facilement (soit seuls, soit en groupe) et ils constituent donc un public potentiel important pour les musées.

Les données démographiques confirment la place de plus en plus grande, d'un point de vue quantitatif, occupée par les seniors. Tous les pays occidentaux et industrialisés sont confrontés à ce phénomène. En France, alors que les plus de 60 ans représentent aujourd'hui environ 12,5 millions de personnes, les projections démographiques les plus récentes de l'INSEE prévoient qu'ils seront 21 millions en 2035. Cet aspect quantitatif est loin d'être négligeable puisqu'il augure un « boom » important au niveau de la consommation culturelle des seniors, et ce d'autant plus que les futurs seniors sont déjà des consommateurs de loisirs et de produits culturels.

Une autre composante importante est celle touchant aux revenus des seniors : de façon générale, ceux-ci sont et seront dans l'ensemble assez confortables, la maison étant déjà payée et les enfants étant élevés et prêts à entrer dans la vie active, ou s'y trouvant déjà. La génération des baby-boomers est, quant à elle, particulièrement " privilégiée » car plus aisée que les précédentes, aisance liée à des niveaux de qualification plus élevés et à un travail féminin plus développé.

Outre des ressources financières satisfaisantes, les seniors disposent enfin de temps. Dans une société où il est habituel de disposer soit de temps mais pas toujours de ressources suffisantes (chômage), soit de revenus confortables mais pas de temps suffisant pour en profiter, les seniors, eux, disposent des deux (2)

Satisfaits dans beaucoup de domaines, les seniors envisagent les prochaines années avec optimisme.
Selon une enquête réalisée en 2005, ils sont 68 \% en France à attendre et à espérer de grandes améliorations dans leurs loisirs. Beaucoup aspirent en effet à réaliser des rêves qu'ils n'ont pu réaliser avant, notamment voyager ou explorer de nouveaux domaines de connaissance. Beaucoup moins matérialistes que les autres générations, ils sont par contre beaucoup plus sensibles aux valeurs spirituelles, sociales et intellectuelles. En effet, les seniors n'ont plus guère de besoins matériels, mais compte tenu de leurs possibilités financières, ils peuvent et veulent investir dans des biens « immatériels » qui répondent mieux à leurs besoins d'autoréalisation, de socialisation, de découverte et de plaisir. Cette demande a déjà généré une offre de la part des touropérateurs comme des universités, qui ont très vite saisi l'intérêt qu'ils pouvaient retirer en créant des produits spécifiques pour ce groupe d'âge. Il s'agit là d'une donnée clé pour les musées, sachant que ces derniers participent autant au secteur des loisirs qu'à celui de l'éducation informelle.

\section{Pratiques culturelles des seniors}

d'aujourd'hui et de demain

Plusieurs éléments permettent de qualifier la culture des seniors : il s'agit tout d'abord d'une culture active qui exclut toute idée d'accumulation. Deuxièmement, elle ne fonctionne pas sur le registre de la consommation, ce qui implique que son contenu importe plus que le contenant. Troisièmement, elle est un pont entre le passé et l'avenir : comme l'histoire dans sa définition large, la culture est ce qui permet d'appréhender le présent à partir du passé, afin de mieux comprendre le futur. Enfin, elle est un instrument qui permet de communiquer non

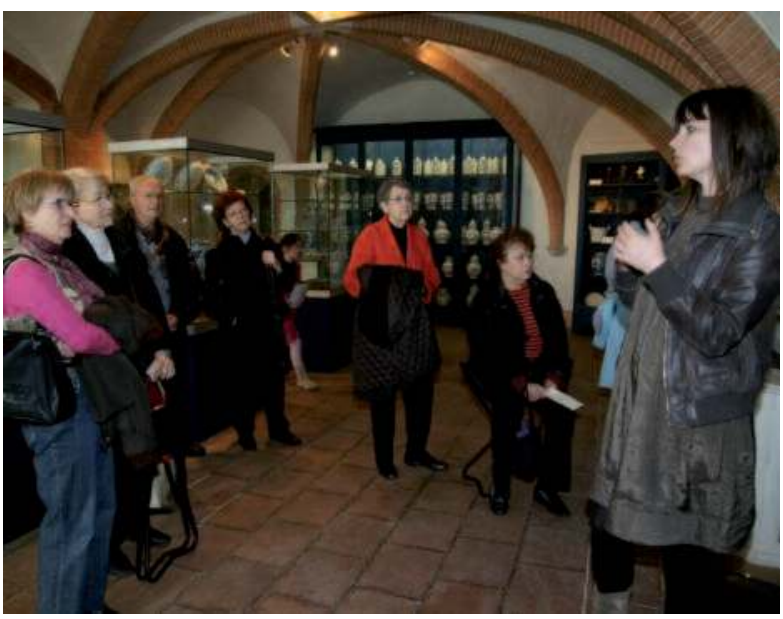

Une visite animée pour un petit groupe permet échanges, découvertes et apprentissage dans des conditions optimales. (c) Musée Ingres, Montauban/Jacques Décatoire (La Dépêche du Midi) 
seulement avec l'extérieur, les autres, mais aussi avec soi-même. Quoi qu'il en soit, la culture ne vaut en elle-même que par les prolongements qu'elle implique, et pas seulement pour elle-même : elle constitue une ouverture, non seulement intellectuelle, mais aussi psychologique et physiologique.

Selon la typologie proposée par Olivier Donnat (3), les seniors se rattachent à l'univers cultivé classique, univers organisé autour d'un axe lecture-patrimoine qui reste à l'écart du «boom musical » et de certaines nouvelles formes d'expression comme l'art contemporain. Les seniors apparaissent tout d'abord comme de grands consommateurs de télévision et de grands lecteurs. Pour ce qui est des pratiques culturelles exercées en dehors de la maison, les musées (avec $23 \%$ ), les monuments historiques $(22 \%)$ et les expositions temporaires (15\%) arrivent dans les sept premières places pour les activités pratiquées par les plus de 60 ans. Les pourcentages touchant aux 40-59 ans placent les activités patrimoniales dans les huit premiers rangs avec des pourcentages particulièrement intéressants pour l'avenir : $30 \%$ pour les musées, $29 \%$ pour les monuments historiques et $24 \%$ pour les expositions temporaires. Par ailleurs, toutes les données quantitatives sont en hausse depuis les années quatre-vingt, positionnant peu à peu la catégorie des seniors dans la moyenne nationale.

Associée aux données démographiques et économiques, si une telle évolution se poursuit, on comprend aisément la place qu'occuperont les seniors dans le public des musées ou autres sites patrimoniaux, d'autant qu'ils disposeront, contrairement à leurs prédécesseurs, des clés permettant l'accès à la "culture de sorties » (Olivier Donnat), à savoir : des revenus suffisants, un héritage culturel plus important, un capital social et surtout relationnel plus étoffé, un niveau de diplômes plus élevé et surtout une mobilité accrue (automobilité).

Compte tenu de la rareté des données disponibles, il est difficile aujourd'hui de brosser une typologie complète des musées ayant les faveurs des seniors : on peut seulement noter que les femmes sont davantage présentes dans les musées des beaux-arts et les hommes dans ceux consacrés à l'histoire. De façon plus générale, les 60 ans et plus accordent leur faveur au patrimoine historique et religieux ainsi qu'aux musées de société, alors que la génération suivante, soit les 40-59 ans, est plus assidue dans les musées consacrés aux beaux-arts.
Le senior en tant que visiteur de musée : éléments pour un premier constat

Il existe trois grandes catégories de visiteurs adultes, et donc seniors : les visiteurs de type cognitif, les visiteurs de type émotif et les visiteurs de type sensitif (4).

Les premiers privilégient les connaissances et les explications, un de leurs plaisirs étant de comprendre ce qu'ils voient en cherchant souvent à obtenir le maximum d'informations. Les seconds privilégient surtout les valeurs véhiculées par ce qu'ils voient : ils aiment évoquer, raconter et s'enthousiasmer, car pour eux c'est communiquer qui est important. Quant aux troisièmes, ils privilégient l'apprentissage par l'action : ils aiment avant tout toucher et manipuler, afin de mieux comprendre le fonctionnement et la fabrication de ce qu'ils voient. Ces différentes attitudes ne sont pas exclusives entre elles et peuvent même cohabiter dans des proportions variables chez un même individu. Néanmoins il ressort des différentes enquêtes que le cognitif domine $(63 \%)$ par rapport au sensitif et à l'émotif, qui se partagent de façon à peu près équivalente les $37 \%$ restants.

Même s'il est important - et le point de départ de tout travail - l'artefact n'est pas tout. Il peut être prétexte à d'autres développements de la personnalité du visiteur, au niveau social, psychologique, moral... et apporter d'autres bénéfices que de nouvelles connaissances ou un plaisir purement intellectuel. Comprendre cet état de fait permet de tempérer le pessimisme de certains guides à l'encontre du public des seniors, et par là même de mieux saisir le rôle social du musée. On retrouve, une fois de plus, un élément clé dans ce que peut apporter le musée : le plaisir, ou plus exactement une série de plaisirs.

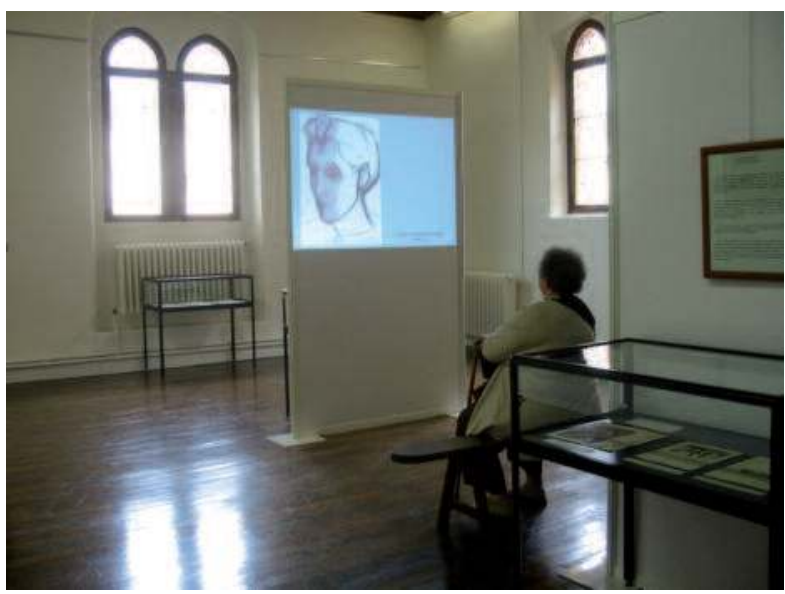

L'importance des aménagements en termes de circulation et de repos ๑) Musée d'Albertville/Régine Monod 
Comme le souligne Bernard Lefebvre, le « loisir culturel [anquel participe le musée] est l'utilisation facultative du temps libre dans un but de divertissement, d'évasion, de culture, d'instruction, en vue de la réalisation de soi... Il implique toujours la recherche $d u$ plaisir et aboutit la plupart du temps à une sensation de bien-être général ».

Suite aux travaux menés outre-atlantique, il ressort que les visiteurs seniors réalisent les opérations suivantes : premièrement, constater, identifier, expliquer ; deuxièmement, saisir, vérifier, associer, et enfin comparer, résoudre, suggérer. $67 \%$ des interrogations portent sur l'objet muséal et seulement $17 \%$ sur les modalités de la recherche ou $9 \%$ sur la présentation muséographique. L'objet reste donc au centre non seulement de la visite, mais aussi du questionnement du visiteur. Au niveau des artefacts, $40 \%$ des questions portent sur ses caractéristiques visibles et $60 \%$ sur ses caractéristiques non visibles. L'intérêt du visiteur pour ce qu'il observe est donc beaucoup moins superficiel qu'il n'y paraît et dépasse largement la simple observation ou contemplation par un engagement intellectuel réel.

Quant aux attentes des seniors vis-à-vis de l'institution muséale, elles ont été clairement identifiées : apprendre et découvrir, réutiliser et/ou réinvestir un bagage d'expériences et de valeurs et enfin être libre. La liberté est un élément très apprécié, s'agissant moins de la liberté vis-à-vis du programme éducatif proposé par le musée que de la liberté intellectuelle. Le musée se doit de favoriser et d'accepter la réceptivité personnelle face à l'artefact observé. Il doit d'ailleurs encourager la représentation personnelle que le visiteur se construit car elle participe activement au développement de la confiance que celui-ci peut avoir de lui-même. Ceci constitue la première étape pour apprendre à observer, à discuter et à juger par soi-même. Ainsi, lors de visites guidées par exemple, il faut se garder de faire intervenir trop tôt des explications et privilégier une approche culturelle large, portant sur le cadre historique auquel appartient l'objet étudié. Le discours tenu doit viser plus à la recontextualisation qu'à l'étude proprement dite de l'objet, du moins dans un premier temps. Une telle liberté peut aussi favoriser chez le visiteur le développement de sa sensibilité esthétique et de sa capacité à analyser les artefacts présentés.

\section{Pour une rencontre réussie}

\section{L'éducation muséale et les seniors}

Un programme éducatif peut constituer un stimulant appréciable pour les seniors, tant au plan intellectuel

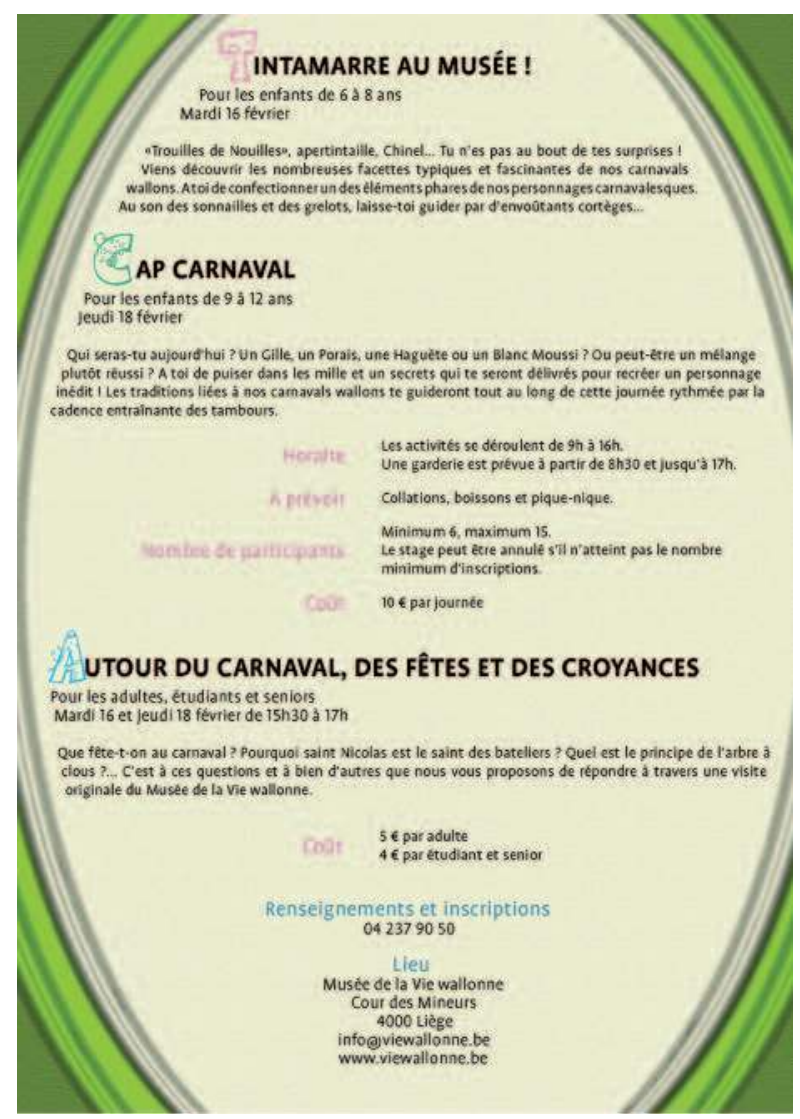

La programmation 2010 du musée de la Vie wallonne de Liège intègre les seniors sans les « ghettoïser ». () Musée de la Vie wallonne

qu'affectif. Il constitue non seulement l'occasion d'acquérir de nouvelles connaissances, mais offre aussi un volet social valorisant, laissant place aux émotions, aux souvenirs, aux questions, à la réflexion, à la curiosité et surtout au plaisir.

En dehors du triplé apprentissage/motivation/observation qui fonde toute éducation muséale et qui reste valable pour cette catégorie de public, l'action muséale en direction des seniors doit tenir compte d'un certain nombre d'éléments, le vieillissement amenant diverses modifications biologiques, physiologiques, émotives et intellectuelles.

Laccueil doit être particulièrement soigné. Il conviendra de donner immédiatement les informations pratiques essentielles (toilettes, vestiaires, cafétéria) et de les informer sur ce qu'ils vont voir et sur les objectifs de l'exposition, ce qui les rendra plus détendus et donc plus réceptifs.

Dans le cas d'une visite guidée de groupe, il est important de faire attention à ne pas adopter un rythme trop rapide, et de prévoir des pauses (avec pourquoi pas, une pause café, toujours appréciée des seniors). Au 
niveau du contenu du programme, il conviendra de trouver un équilibre entre l'intérêt populaire et le contenu académique, en fournissant une visite solide et bien organisée. Quel que soit le thème traité, l'approche devra être multidisciplinaire et prendre impérativement appui sur l'exposition ou des éléments constitutifs de celle-ci. L'approche doit enfin se faire dans une atmosphère agréable et détendue, libre et légère, loin de toute démarche scolaire. Elle doit être sérieuse, tout en refusant de «se prendre au sérieux ».

En complément au programme distribué, beaucoup de seniors souhaiteraient avoir la possibilité de recourir à une brochure explicative comportant des reproductions. À ce niveau comme pour tout document écrit, il conviendra de veiller aux éléments suivants : la taille minimum des caractères doit être de douze points, la police de caractères sobre et claire ; il faut éviter le lettrage inversé et préférer la formule des caractères foncés sur fond pâle, utiliser du papier et de l'encre mats, aérer le texte et la présentation, privilégier des textes courts et concis, et enfin éviter toute présentation «fantaisiste » en conservant une mise en page très classique.

\section{Les apports de la gérontagogie (5)}

Grâce à l'ouverture relativement ancienne de l'université au monde des seniors, l'enseignement est à même d'apporter aux musées quelques clés quant au mécanisme d'apprentissage des seniors.

Il est indéniable que l'âge affecte les capacités d'apprendre, mais de façon beaucoup moins prononcée que ne le laissaient supposer les premières études sur le sujet. Identifiés dès 1967 par Botwinick (6), les aspects cognitifs s'articulent autour de quatre domaines : la mémoire, l'intelligence, l'apprentissage et les fonctions d'intégration.

Au niveau de la mémoire, seule celle à court terme est affectée par l'âge, celle à long terme ne l'étant pas du tout : les seniors prennent en réalité plus de temps à mémoriser les nouvelles données.

Pour ce qui est de l'intelligence, elle n'est pas affectée par l'âge. Seule la créativité a tendance à baisser, tandis que la capacité à utiliser d'anciennes connaissances ou expériences pour résoudre un problème donné, continue à se développer.

Si l'âge n'interrompt pas les capacités d'apprentissage, il en affecte cependant la rapidité, d'où la nécessité d'accorder au senior le temps dont il a besoin pour apprendre.

Quant aux fonctions d'intégration, elles ont tendance à décliner, qu'il s'agisse de la formation de concepts, de la solution de problèmes ou encore de la créativité. Il ressort qu'au niveau cognitif toutes les fonctions importantes se maintiennent jusqu'à un âge avancé, et que les seniors disposent en outre d'un atout non négligeable : ils ont tendance à contextualiser les savoirs abstraits, à résumer une histoire en essayant de voir les implications sociales et morales plutôt que les détails, et à resituer les problèmes dans une perspective sociale, affective et parfois personnelle.

Quand les seniors apprennent, il s'agit d'une démarche ouverte, désintéressée, sans lien avec la productivité. Le but d'une telle éducation est l'enrichissement, qui passe par un accomplissement de soi et la possibilité d'une participation active à la vie politique, sociale et culturelle. André Lemieux parle d'une " actualisation de soi » chez les seniors.

Un des points fondamentaux mis en avant par tous les chercheurs est la nécessité de prendre comme point de départ et de mettre à profit les acquis et l'expérience des seniors. Il faut donc dépasser le stade de l'information, qui par nature reste superficielle et non intégrée (oubli), pour atteindre ceux du savoir et de la connaissance qui, parce que liés à des savoirs et des acquis antérieurs, vont être conservés. Dialogue, échanges, interactivité avec un formateur, mais aussi entre les seniors vont permettre une participation active et personnelle et donc favoriser l’intégration des nouvelles connaissances.

Au niveau des programmes destinés à des seniors, il est préconisé un contenu de haute qualité (exigence élevée de ce public compte tenu de l'élévation constante de son niveau de formation) ; une durée de 45 minutes maximum (l'attention baissant plus rapidement avec l'âge) avec une alternance de temps forts qui nécessitent une attention soutenue et de temps moins denses qui permettent à l'esprit d'être moins sollicité ; une organisation claire (pas d'intervention improvisée, mais quelque chose de bien «charpenté » qui permet toutefois le dialogue et les échanges) et un recours aux ressources du groupe les seniors apprennent mieux avec et par les autres, car ils sont très sensibles aux expériences vécues avec plusieurs personnes. Doivent aussi être au rendez-vous : la sollicitude et la convivialité (naturelles et non artificielles), les notions de découverte et le plaisir (l'intervention doit laisser une large part à la découverte et à la conscience critique). 


\section{Notes}

(1) D’après le diagramme réalisé par Alan Chapman à partir des travaux de Maslow in www.businessballs.com/maslow.htm

(2) Poquet G. Le pouvoir et le rôle économique des plus de 50 ans. Paris : CREDOC, 1996, 80 p.

(3) Donnat O. Regards croisés sur les pratiques culturelles. Paris : La Documentation française, 2003, 348 p.

(4) D'après les travaux de Colette Dufresne-Tassé et d'André Lefebvre, voir en bibliographie.

(5) Terme qui trouve son origine dans les travaux d'André Lemieux et qui désigne la pédagogie touchant à l'éducation des personnes âgées ou seniors.

(6) Botwinick J. Cognitive processes in maturity and old ages. New-York, : Springer, 1967

\section{Bibliographie}

Allard, M. et Lefebvre, B. Musée, culture et éducation. Montréal : GREM, 2000, 197 p.

Donnat, O. Les pratiques culturelles des Français à l'ère numérique. Paris, La Découverte /La Documentation française, 2009, 292 p.

Dufresne-Tassé, C. et Lefebvre, A. Psychologie du visiteur de musée Contribution à l'éducation des adultes en milieu muséal. Montréal : Hurtubise, 1996, 174 p.
Hooper-Greenhill, E. Museums and education : purpose, pedagogy, performance. Londres : Routledge, 2007, 231 p.

Howard-Falk, J. Learning from museums : visitor experiences and the making of meaning. Walnut Creek : AltaMira Press, 2000, 272 p.

Lamdin, L. Elderlearning - New Frontier in an aging society. Phoenix : American Council on Education, 1997, 203 p.

Lefebvre, B. Les aînés et la fréquentation des musées, Cahier du GREM, $\mathrm{n}^{\circ} 2,1998,64 \mathrm{p}$.

Lefebvre, H. Étude descriptive des bénéfices d'une visite au musée pour des personnes âgées. Thèse de doctorat, UDM, 1994, 190 p.

Lemieux, A. Gerontagogy beyond words : a reality. Montréal : Éditions Nouvelles, 2005, $223 \mathrm{p}$.

Martha-Tyler, J. Geragogy : a theory for teaching the Elderly. New York, 1988, $156 \mathrm{p}$

Niace Museums and Adults learning : perspectives from Europe. Leicester, 2000, 242 p.

Serrière, F. Conquérir le marché des baby-boomers. Paris : Pearson Education France, 2006, 203 p. 